\title{
RANKL: A promising circulating marker for bone metastasis response
}

\author{
TONI IBRAHIM ${ }^{1}$, MARIANNA RICCI ${ }^{1}$, EMANUELA SCARPI ${ }^{2}$, ALBERTO BONGIOVANNI $^{1}$, ROSSANA RICCI ${ }^{1}$, \\ NADA RIVA $^{1}$, CHIARA LIVERANI ${ }^{1}$, ALESSANDRO DE VITA ${ }^{1}$, FEDERICO LA MANNA ${ }^{1}$, DEVIL OBOLDI $^{3}$, \\ PATRIZIA SERRA ${ }^{2}$, FLAVIA FOCA ${ }^{2}$, LORENZO CECCONETTO ${ }^{4}$, DINO AMADORI ${ }^{4}$ and LAURA MERCATALI ${ }^{1}$ \\ ${ }^{1}$ Osteoncology and Rare Tumors Center; \\ ${ }^{2}$ Unit of Biostatistics and Clinical Trials; ${ }^{3}$ Radiology Unit; ${ }^{4}$ Department of Medical Oncology, \\ Istituto Scientifico Romagnolo per lo Studio e la Cura dei Tumori (IRST) IRCCS, I-47014 Meldola, Italy
}

Received July 1, 2015; Accepted July 20, 2016

DOI: 10.3892/ol.2016.4977

\begin{abstract}
Bone metastases are a frequent event in patients with solid tumors. Although great advances have been made in the treatment of these patients, the identification of novel, accurate indicators of bone response would greatly facilitate the clinical management of the disease. The receptor activator of nuclear factor- $\kappa \mathrm{B}$ (RANK)/RANK ligand (RANKL)/osteoprotegerin (OPG) signaling pathway is significantly involved in bone metastasis formation. The main aim of the present study was to evaluate the role of circulating RANK, RANKL and OPG levels in predicting bone response. Marker accuracy was also compared with that of the conventional tumor marker N-terminal telopeptide of type I collagen (NTX). A prospective study was performed on 49 patients with bone metastases from breast, lung and prostate cancer, who were undergoing treatment with zoledronic acid. Patients were monitored for 1 year with blood tests, clinical evaluation and instrumental exams according to the response evaluation criteria of the University of Texas M. D. Anderson Cancer Center (Houston, TX, USA) and the Positron Emission Tomography Response Criteria in Solid Tumors. Circulating RANK/RANKL/OPG transcripts and NTX levels were evaluated by reverse transcription -quantitative polymerase chain reaction and immune enzymatic assay, respectively. The baseline RANKL levels differed significantly between responders and non-responders, whereas no differences in NTX levels were observed between the two groups. Receiver operating characteristic curve evaluation for all markers revealed that RANKL was the most accurate marker, with an area under the curve of 0.74 (95\% confidence interval, 0.54-0.93). In addition, RANKL, which is the target of the novel monoclonal antibody denosumab, was the most accurate predictor of bone response in the present series of patients with
\end{abstract}

Correspondence to: Dr Laura Mercatali, Osteoncology and Rare Tumors Center, Istituto Scientifico Romagnolo per lo Studio e la Cura dei Tumori (IRST) IRCCS, 40 Via Maroncelli, I-47014 Meldola, Italy E-mail: laura.mercatali@irst.emr.it

Key words: RANK, RANKL, OPG, NTX, bone metastases bone metastases. Thus, the use of RANKL as a marker could potentially improve clinical practice, as current bone response evaluation is still somewhat problematic.

\section{Introduction}

Bone metastases are common in numerous solid cancers, including breast, prostate and lung cancer $(1,2)$. In the USA, $\sim 2 / 3$ of patients who succumb to cancer each year have bone metastases, and $20-25 \%$ of patients with neoplastic disease develop clinically evident bone metastases during the natural course of the disease $(3,4)$.

Bone metastases are responsible for high morbidity and reduced quality of life due to the frequent onset of clinical complications defined as skeletal-related events (SREs), which lead to a reduction in functional independence and quality of life, decrease survival rates and substantially increase healthcare costs $(5,6)$.

Bisphosphonates have improved the quality of life of patients with bone metastases from breast cancer by inducing both a reduction in SREs and in the risk of mortality (7). In particular, zoledronic acid (ZA), a potent third-generation nitrogen-containing bisphosphonate, has achieved widespread clinical use in the treatment of bone metastases from solid tumors (8-10). Denosumab, a monoclonal antibody against RANKL, has been demonstrated to be superior to ZA in delaying or preventing SREs (11).

Despite the clinical improvements, bone response evaluation remains a critical problematic area (12). In fact, radiological assessment of bone metastasis response is often controversial, and novel response evaluation criteria have been implemented in recent years, including that of the University of Texas M. D. Anderson Cancer Center (MDA; Houston, TX, USA) and the Positron Emission Tomography Response Criteria in Solid Tumors (PERCIST) (12). Another potentially useful tool to aid clinicians in measuring bone response could be the use of circulating predictive markers. Although numerous markers have been evaluated for this purpose, including cross-linked C-terminal telopeptides of type I collagen (ICTP) and cross-linked $\mathrm{N}$-terminal telopeptides of type I collagen (NTX), no really accurate markers have been identified to 
date (13-17). The present authors previously focused their attention on the molecules of the receptor activator of nuclear factor- $\kappa \mathrm{B}$ (RANK)/RANK ligand (RANKL)/osteoprotegerin (OPG) axis that govern bone resorption and are involved in bone metastasis $(18,19)$. This axis is also probably involved in the early development of metastases (18).

Briefly, RANKL binds and activates its receptor RANK on the surface of osteoclasts, thus stimulating osteoclast differentiation and maturation, which leads to an increase in bone resorption (20). OPG, by binding to RANKL, acts as a decoy receptor, thus inhibiting osteoclastogenesis (20).

The primary aim of the present study was to evaluate RANKL, RANK and OPG as predictive markers of response to bone-targeted therapy in patients with bone metastases. Secondary aims were to compare the RANKL, RANK and OPG results with those obtained from NTX and carcinoembryonic antigen (CEA)/carbohydrate antigen (CA)153 assessment in terms of their accuracy in predicting bone response during treatment.

\section{Materials and methods}

Study design. The present study was a prospective study conducted at Istituto Scientifico Romagnolo per lo Studio e la Cura dei Tumori (IRST) IRCCS (Meldola, Italy). The study protocol was approved by the local ethics committee in accordance with the ethical standards of the 1964 Declaration of Helsinki. All patients were enrolled between March 2007 and December 2009, and provided informed written consent to take part in the study.

Case series. Peripheral venous blood (PB) samples were obtained from 49 consecutive patients with bone metastases from solid tumors. Of these, 36 patients had breast cancer, 7 had prostate cancer and 6 had lung cancer. The eligibility criteria were as follows: Males and females aged $\geq 18$ years; histological confirmation of solid tumor and radiological confirmation of bone metastases; previous hormone therapy or chemotherapy, alone or in combination with biological agents such as trastuzumab; life expectancy $>6$ months; good performance status (PS) according to the Eastern Cooperative Oncology Group (ECOG; http://ecog-acrin. org/resources/ecog-performance-status); normal hepatic function with total bilirubin $<2 \mathrm{mg} / \mathrm{dl}$; and serum creatinine $<2 \mathrm{mg} / \mathrm{dl}$. Exclusion criteria were as follows: History of postmenopausal osteoporosis; administration of estrogen, calcitonin, vitamin D or calcium supplements prior to enrollment; prior therapy with bisphosphonates; osteonecrosis of the jaw; or dental conditions requiring surgery to the oral cavity.

Treatment and follow-up. All patients received $4 \mathrm{mg}$ of ZA (Zometa ${ }^{\circledR}$; Novartis International AG, Basel, Switzerland) in $100 \mathrm{ml}$ of $0.9 \%$ saline solution over $15 \mathrm{~min}$ as an intravenous infusion every 28 days. Follow-up comprised blood tests and instrumental exams according to PERCIST and the MDA response evaluation criteria (12), which were conducted every 3 or 4 months after the diagnosis of bone metastases for a maximum of 12 months.

Biological samples. PAXgene Blood RNA Tubes (PreAnalytiX; BD Biosciences, Franklin Lakes, NJ, USA) were stored at $-80^{\circ} \mathrm{C}$ until RNA extraction. PB samples were centrifuged at $2,000 \mathrm{x} \mathrm{g}$ for $15 \mathrm{~min}$, and stored at $-80^{\circ} \mathrm{C}$.

NTX immunoassays. NTX levels were measured using a competitive-inhibition enzyme-linked immunosorbent assay in accordance with the manufacturer's protocol (19).

RANK/RANKL/OPG. Blood RNA was extracted with the PAXgene Blood RNA kit (Qiagen, Hilden, Germany) according to the manufacturer's protocol. A total of $500 \mathrm{ng}$ of RNA were reverse transcribed using the iScript cDNA Synthesis kit (Bio-Rad Laboratories, Inc., Hercules, CA, USA). Reverse transcription-quantitative polymerase chain reaction was performed as previously described (19).

Statistical analysis. The primary and secondary objectives of the current study were to evaluate the predictive role of different circulating markers and to assess bone metastasis status, respectively. Thus, only patients for whom an objective assessment of tumor response was feasible were included in the analysis. The Shapiro-Wilk test was used to test the normality distribution of each biomarker. As a normal distribution was not observed, the natural logarithm of the original values of each biomarker was used for the main analyses. For the primary objective, data at baseline were analyzed by a logistic regression model. Non-parametric ranking statistics (median test) were used to analyze the association between the serum levels of each marker, considered as continuous variables, and bone metastasis status or patient characteristics.

The present study evaluated whether marker levels differed between patients with stable disease or with partial response (PR)/complete response (CR) (responders) and those with progressive disease (non-responders), as defined according to the MDA response evaluation criteria and PERCIST. Marker accuracy was compared by receiver operating characteristic (ROC) curve analysis, in which the marker values were coupled with the best bone response (Fig. 1). The area under the curve (AUC), as well as the positive predictive values (PPVs) and negative predictive values (NPVs) of bone progression were calculated for each circulating marker. The PPVs and NPVs of each marker were defined on the basis of $\geq 25 \%$ marker variations with respect to the corresponding baseline values and to bone response. Statistical analyses were performed with SAS statistical software version 9.3 (SAS Institute, Cary, NC, USA). A P-value of $<0.05$ was considered to indicate a statistically significant difference.

\section{Results}

Patients characteristics. The median age of patients was 62 years (range, 34-86 years); there were 37 females, of whom, $77.8 \%$ were post-menopausal, and 12 males. In total, $82 \%$ of patients had ECOG PS 0, 16\% had ECOG PS 1 and 2\% had ECOG PS 2 at the start of the study. With regard to the biopathological features of the primary breast cancers, ductal histology was the most frequent (58\%), and 92 and $66.7 \%$ of primary tumors were estrogen- and progesterone-positive, respectively. The Ki-67 proliferative index was high in $66.7 \%$ of patients, and human epidermal growth factor receptor 2 was amplified in $36.1 \%$ of patients. A total of 9 and $65 \%$ of patients 
Table I. Predictive power of baseline values with respect to the best bone response (logistic regression).

Overall case series, responders vs. non-responders
Breast cancer subgroup, responders vs. non-responders

\begin{tabular}{lcccr}
\cline { 2 - 3 } Marker & OR $(95 \% \mathrm{CI})$ & P-value & OR $(95 \% \mathrm{CI})$ & P-value \\
\hline RANK & $1.60(0.70-3.69)$ & 0.274 & $1.44(0.57-3.64)$ & 0.441 \\
RANKL & $2.55(0.95-6.86)$ & 0.063 & $3.20(0.79-13.08)$ & 0.105 \\
OPG & $1.02(0.67-1.56)$ & 0.914 & $0.95(0.52-1.75)$ & 0.870 \\
RANKL/OPG & $1.25(0.76-2.04)$ & 0.380 & $1.47(0.72-3.01)$ & 0.291 \\
NTX & $1.20(0.27-4.67)$ & 0.878 & $1.63(0.33-8.00)$ & 0.550 \\
CEA & $1.36(0.60-3.11)$ & 0.460 & $1.30(0.54-3.16)$ & 0.557 \\
CA153 & $1.45(0.63-3.31)$ & 0.380 & $1.45(0.63-3.31)$ & 0.380
\end{tabular}

RANK, receptor activator of nuclear factor- $\mathrm{kB}$; RANKL, RANK ligand; OPG, osteoprotegerin; NTX, N-terminal telopeptide of type I collagen; CEA, carcinoembryonic antigen; CA, carbohydrate antigen; OR, odds ratio; CI, confidence interval.

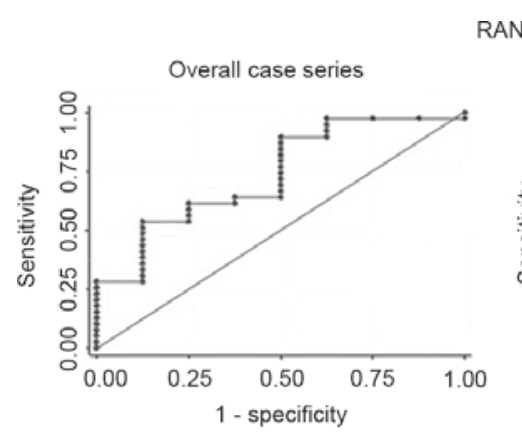

Area under ROC curve $=0.7372$

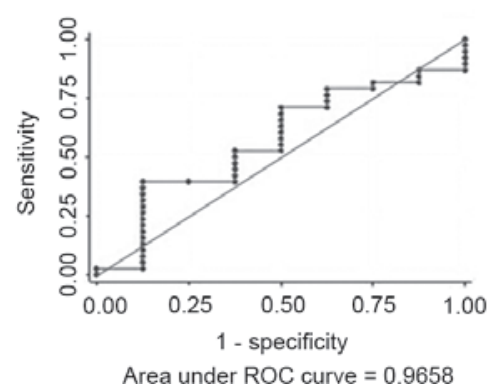

RANKL

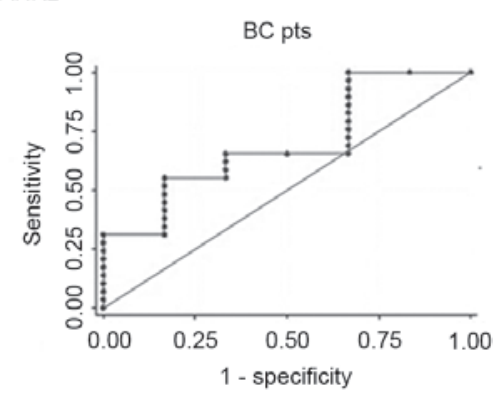

Area under ROC curve $=0.6954$

NTX

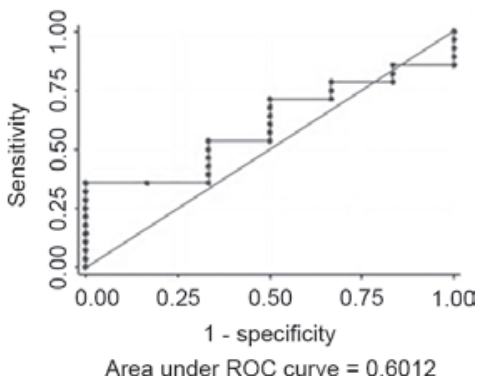

Figure 1. ROC curve analysis of RANKL and NTX. BC, breast cancer; pts, patients; RANKL, receptor activator of nuclear factor- $\kappa$ B ligand; NTX, N-terminal telopeptide of type I collagen; ROC, receiver operating characteristic.

had previously received neoadjuvant or adjuvant therapy, respectively, and $81.2 \%$ had undergone hormone therapy.

In addition, $51 \%$ of patients in the overall case series also had visceral metastases, compared with $42 \%$ in the breast cancer subgroup. Bone metastases were mainly osteolytic (56\% in the overall case series and $59 \%$ in the breast cancer subgroup), and only $10 \%$ of patients (11\% in the case of breast cancer patients) had only one bone lesion when bone disease was diagnosed.

Correlation between markers and objective response. The results of the evaluation of different circulating markers as predictors of response to bone-targeted therapy are reported in Table I. The 1-unit increase in RANKL values improved the probability of responding to therapy by 2.5 -fold in the overall case series. None of the biomarkers was significantly predictive of response to ZA. The median RANKL levels in non-responders were $35 \%$ lower than those in responders $(\mathrm{P}=0.037)$. The NTX values remained unchanged. The variations in other markers were not significant (Table II). ROC curve analysis was performed for all circulating markers to evaluate bone marker accuracy, and the best result was obtained for RANKL, with an AUC of 0.74 [95\% confidence interval (CI), 0.54-0.93] (Fig. 1). CEA and CA15-3 exhibited an AUC of 0.61 (95\% CI, 0.33-0.88) and 0.73 (95\% CI, 0.48-0.99), respectively (Table III).

Considering a $\geq 25 \%$ marker variation with respect to baseline values as a cut-off, the PPV for RANKL was 22.7 (95\% CI, 12.3-37.6), and the NPV was 88.0 (95\% CI, 74.0-95.0). The NTX PPV and NPV were 0.0 (95\% CI, 0.0-9.6) 
Table III. Evaluation of circulating marker accuracy by ROC curve analysis.

\begin{tabular}{lcclcc}
\hline & \multicolumn{2}{c}{$\begin{array}{c}\text { Overall case } \\
\text { series }\end{array}$} & & \multicolumn{2}{c}{$\begin{array}{c}\text { Breast cancer } \\
\text { patients }\end{array}$} \\
\cline { 2 - 3 } \cline { 6 - 7 } Marker & AUC & $95 \%$ CI & & AUC & $95 \%$ CI \\
\hline RANK & 0.58 & $0.35-0.81$ & & 0.61 & $0.37-0.86$ \\
RANKL & 0.74 & $0.54-0.93$ & & 0.70 & $0.48-0.93$ \\
OPG & 0.53 & $0.33-0.72$ & & 0.52 & $0.32-0.72$ \\
RANKL/OPG & 0.60 & $0.41-0.81$ & & 0.64 & $0.44-0.84$ \\
NTX & 0.57 & $0.34-0.79$ & & 0.60 & $0.38-0.82$ \\
CEA & 0.61 & $0.33-0.88$ & & 0.59 & $0.28-0.91$ \\
CA15-3 & 0.73 & $0.48-0.99$ & & 0.73 & $0.48-0.99$
\end{tabular}

AUC, area under the curve; CI, confidence interval; ROC, receiver operating characteristic; RANK, receptor activator of nuclear factor- $\mathrm{kB}$; RANKL, RANK ligand; OPG, osteoprotegerin; NTX, $\mathrm{N}$-terminal telopeptide of type I collagen; CEA, carcinoembryonic antigen; CA, carbohydrate antigen.

and 81.4 (95\% CI, 66.7-90.8), respectively (Table III). Neither the PPV or NPV results improved when circulating markers were considered as dichotomous variables (data not shown).

Breast cancer subgroup. The logistic regression model revealed that a 1-unit increase in RANKL values increased by 3 -fold the probability of responding to therapy in the breast cancer subgroup. Conversely, the same increase in NTX, CEA and CA15-3 values increased the probability of bone response by 63,30 and $45 \%$, respectively.

The median levels of NTX at baseline, similarly to those of CEA and CA15-3, did not differ between responders and non-responders. However, a decrease of 30 and $15 \%$ was observed in OPG and RANKL levels, respectively, in non-responders (Table II). ROC curve analysis of all markers revealed that RANKL and CA15-3 were the two most accurate markers, with an AUC of 0.70 (95\% CI, 0.48-0.93) and 0.73 (95\% CI, 0.48-0.99), respectively (Table III).

The PPVs were $26.7 \%$ (95\% CI, 13.8-44.6\%) for RANK, $21.4 \%$ (95\% CI, 74.0-97.0\%) for RANKL, $13.3 \%$ for OPG (95\% CI, 4.8-29.9\%) and 13.3 (95\% CI, 4.8-29.9) for the ratio RANKL/OPG. The NPV of these markers ranged from 80.0 to 90.0\%. NTX PPV and NPV were $0.0 \%$ (95\% CI, 0.0-12.6\%) and $80.6 \%$ (95\% CI, 62.9-91.4\%), respectively (Table IV). The PPVs of CEA and CA15-3 were $25.0 \%$ (95\% CI, 8.3-52.6\%) and $33.3 \%$ (95\% CI, 13.5-60.4\%), respectively, while their NPVs were $83.3 \%$ (95\% CI, 55.9-96.0\%) and 84.6\% (95\% CI, 57.2-96.6\%), respectively.

Circulating markers and clinicopathological variables. The median concentrations of all markers were independent of age and gender, and did not differ as a function of clinical or pathological features of the primary tumor. Considering only the primary breast cancer subgroup, the OPG median values of patients with osteoblastic lesions were 2-fold higher (4.34 vs. 2.08), albeit not significantly, than those of patients 
Table IV. Evaluation of marker accuracy.

\begin{tabular}{|c|c|c|c|c|}
\hline \multirow[b]{2}{*}{ Marker } & \multicolumn{2}{|c|}{ Overall $(95 \% \mathrm{CI})$} & \multicolumn{2}{|c|}{ Breast cancer patients $(95 \% \mathrm{CI})$} \\
\hline & PPV (\%) & NPV $(\%)$ & PPV (\%) & NPV $(\%)$ \\
\hline RANK & $23.8(13.1-38.8)$ & $88.4(75.0-95.5)$ & $26.7(13.8-44.6)$ & $90.0(74.0-97.0)$ \\
\hline RANKL & $22.7(12.3-37.6)$ & $88.0(74.5-95.2)$ & $21.4(10.0-39.0)$ & $85.7(68.9-94.6)$ \\
\hline OPG & $12.5(5.1-26.1)$ & $80.6(66.0-90.2)$ & $13.3(4.8-29.9)$ & $80.0(62.5-90.9)$ \\
\hline RANKL/OPG & $11.8(4.7-25.2)$ & $80.0(65.3-89.7)$ & $13.3(4.8-29.9)$ & $80.0(62.5-90.9)$ \\
\hline NTX & $0.0(0.0-9.6)$ & $81.4(66.7-90.8)$ & $0.0(0.0-12.6)$ & $80.6(62.9-91.4)$ \\
\hline CEA & N/A & N/A & $25.0(8.3-52.6)$ & $83.3(55.9-96.0)$ \\
\hline CA15-3 & N/A & N/A & $33.3(13.5-60.4)$ & $84.6(57.2-96.6)$ \\
\hline
\end{tabular}

PPV, positive predictive value; NPV, negative predictive value; $\mathrm{CI}$, confidence interval; RANK, receptor activator of nuclear factor- $\mathrm{kB}$; RANKL, RANK ligand; OPG, osteoprotegerin; NTX, N-terminal telopeptide of type I collagen; CEA, carcinoembryonic antigen; CA, carbohydrate antigen; N/A, not applicable.

with osteolytic/mixed lesions ( $\mathrm{P}=0.400)$. Conversely, the NTX median values were almost identical in the two subgroups (15.6 vs. $15.5 \mathrm{nM}$ BCE, respectively). No clear differences were observed for other markers. With regard to the number of bone lesions, patients with an initial diagnosis of only one bone lesion exhibited 2 -fold higher RANKL and RANKL/OPG median values than other patients. In contrast, a significant increase in NTX was observed in patients with $>6$ lesions. A total of 7 SREs occurred in the overall case series during the 12 months of the study. Marker values were not significantly different in patients with or without SREs.

\section{Discussion}

The treatment of patients with bone metastases has markedly improved over the last 10 years in terms of quality of life and survival due to the introduction of bone-targeted therapies $(11,21,22)$. Patients could further benefit from the availability of a more accurate diagnostic tool to evaluate bone response to treatment, as the currently used imaging techniques often produce controversial results $(12,23)$. The aim of the present prospective study was to evaluate the messenger RNA levels of RANK, RANKL and OPG, and the serum levels of NTX, as markers of bone response in patients undergoing treatment with $\mathrm{ZA}$ for bone metastases from different solid tumors. In a previous study, the present authors reported that the median RANKL values decreased by $22 \%$, whereas the median OPG levels increased by $\sim 96 \%$, after 12 months of treatment with ZA (19). In the panel of markers investigated, only NTX exhibited a significant change over time, decreasing by $26 \%$ with respect to baseline levels $(\mathrm{P}<0.0001)(19)$. In the present study, RANKL was demonstrated to be the most accurate marker in predicting bone response, as confirmed by the odds ratios and the AUCs of ROC curves performed for all markers. Furthermore, the median baseline values of RANKL were significantly higher in responders than in non-responders, whereas no changes in NTX levels were observed in the overall case series or the breast cancer subgroup, as previously reported (24). This is a remarkable finding, as NTX is currently one of the most widely used markers to evaluate bone response to treatment in bone metastasis patients (25-27). The urinary NTX levels in patients with bone metastases from solid tumors enrolled in a previous study were analyzed, and the results revealed that the NTX levels were associated with a 3-fold increased risk of SREs in patients with breast cancer (25). In another study, both baseline NTX levels and on-study increased NTX levels were associated with an increased risk of SREs, disease progression and mortality (26). The authors hypothesized that the increase in NTX and bone-specific alkaline phosphatase levels were probably a reflection of tumor growth in bone (26). However, in 2011, the same authors concluded that biochemical markers of bone metabolism reflect ongoing rates of bone resorption and formation in the body as a whole (27), and that they do not provide information on individual lesion sites (27). This finding is in agreement with the present results.

The present study has certain limitations. First, the present case series included patients with different types of primary tumors; however, several other studies on the same topic have also included patients with different primary tumors in their case series $(15,25-28)$. Secondly, our primary end point was objective response according to the MDA response evaluation criteria and PERCIST, whereas phase III clinical trials on ZA and studies on tumor markers tend to have SREs as the clinical endpoint (25-27). The present study opted for objective response since, as reported in other recent studies, only 7 SREs occurred in the overall case series during the entire study period, which were insufficient to perform statistical analyses. To the best of our knowledge, the present is the first study to use the MDA response evaluation criteria and PERCIST together to assess bone response. It also differs from other studies (25-27) in that NTX was evaluated in serum, not in urine. Notably, a recent study prospectively evaluating NTX as a diagnostic marker of bone metastases concluded that these two biological fluids have equivalent sensitivity and specificity (29). In addition to NTX, numerous other markers have been investigated, including bone-specific alkaline phosphatase (bone formation), as well as tartrate-resistant acid phosphatase isoform 5b and CTX (bone remodeling) $(15,27,29)$. Mountzious et al $(15)$ prospectively evaluated RANKL, OPG and CTX, and observed that 
none of these markers was capable of predicting response to bone. In a recent prospective study, Aktas et al observed that procollagen type I N propeptide and ICTP were the best indicators of bone disease respect to tumor routine markers for monitoring response to bisphosphonates in breast cancer patients (14).

In conclusion, the present results identified RANKL as a promising marker of bone metastasis response in patients treated with ZA. RANKL was observed to be more accurate than conventional markers in the breast cancer subgroup, and was a better predictor of bone progression than NTX. These data suggest that the RANKL could serve as an accurate marker of bone response in metastatic patients. High RANKL levels may identify patients with a shift in bone homeostasis towards bone resorption who could benefit from bone-targeted treatment aimed at inhibiting osteoclast action. The present RANKL result is promising, particularly since RANKL is the target of denosumab, which has been recently introduced into clinical practice.

\section{Acknowledgements}

The authors thank Ms. Ursula Elbling of the Istituto Scientifico Romagnolo per lo Studio e la Cura dei Tumori (IRST) IRCCS (Meldola, Italy) for editing the present manuscript. The preliminary results from the current study were presented at The 4th Joint Meeting of ECTS and IBMS, held in Rotterdam, Netherlands, from 25-28 April, 2015.

\section{References}

1. Jemal A, Siegel R, Ward E, Hao Y, Xu J and Thun MJ: Cancer statistics, 2009. CA Cancer J Clin 59: 225-249, 2009.

2. Harries M, Taylor A, Holmberg L, Agbaje O, Garmo H, Kabilan S and Purushotham A: Incidence of bone metastases and survival after a diagnosis of bone metastases in breast cancer patients. Cancer Epidemiol 38: 427-434, 2014.

3. Ibrahim T, Mercatali L and Amadori D: Bone and cancer: The osteoncology. Clin Cases Miner Bone Metab 10: 121-123, 2013.

4. Ibrahim T, Mercatali L, Casadei R and Sabbatini R: Clinical manifestation. In: Osteoncology textbook. Amadori D, Cascinu S, Conte P and Ibrahim T: (eds). Poletto editore, Milan, pp258-pp276, 2010.

5. Mundy GR: Metastasis to bone: Causes, consequences and therapeutic opportunities. Nat Rev Cancer 2: 584-593, 2002.

6. Ibrahim T, Flamini E, Fabbri L, Serra P, Mercatali L, Ricci R, Sacanna E, Falasconi MC, Casadei R, Galassi R, et al: Multidisciplinary approach to the treatment of bone metastases: Osteo-oncology center, a new organizational model. Tumori 95: 291-297, 2009.

7. Lipton A, Theriault RL, Hortobagyi GN, Simeone J, Knight RD, Mellars K, Reitsma DJ, Heffernan M and Seaman J: Pamidronate prevents skeletal complications and its effective palliative treatment in women with breast carcinoma and osteolytic bone metastases: Long term follow-up of two randomized, placebo-controlled trials. Cancer 88: 1082-1090, 2000.

8. Saad F: Clinical benefit of zoledronic acid for the prevention of skeletal complications in advanced prostate cancer. Clin Prostate Cancer 4: 31-37, 2005.

9. Berenson JR, Rosen LS, Howell A, Porter L, Coleman RE, Morley W, Dreicer R, Kuross SA, Lipton A and Seaman JJ: Zoledronic acid reduces skeletal-related events in patients with osteolytic metastases. Cancer 91: 1191-2000, 2001.

10. Amadori D, Aglietta M, Alessi B, Gianni L, Ibrahim T, Farina G, Gaion F, Bertoldo F, Santini D, Rondena R, et al: Efficacy and safety of 12-weekly versus 4-weekly zoledronic acid for prolonged treatment of patients with bone metastases from breast cancer (ZOOM): A phase 3, open-label, randomised, non-inferiority trial. Lancet Oncol 14: 663-670, 2013.
11. Lipton A, Fizazi K, Stopeck AT, Henry DH, Brown JE, Yardley DA, Richardson GE, Siena S, Maroto P, Clemens M, et al: Superiority of denosumab to zoledronic acid for prevention of skeletal-related events: A combined analysis of 3 pivotal, randomised, phase 3 trials. Eur J Cancer 48: 3082-3092, 2012.

12. Costelloe CM, Chuang HH, Madewell JE and Ueno NT: Cancer response criteria and bone metastases: RECIST 1.1, MDA and PERCIST. J Cancer 1: 80-92, 2010.

13. Coleman R, Brown J, Terpos E, Lipton A, Smith MR, Cook R and Major P: Bone markers and their prognostic value in metastatic bone disease: Clinical evidence and future directions. Cancer Treat Rev 34: 629-639, 2008

14. Aktas B, Kasimir-Bauer S, Lehmann N, Kimmig R and Tewes M: Validity of bone marker measurements for monitoring response to bisphosphonate therapy with zoledronic acid in metastatic breast cancer. Oncol Rep 30: 441-447, 2013.

15. Mountzios G, Terpos E, Syrigos K, Papadimitriou C, Papadopoulos G, Bamias A, Mavrikakis M and Dimopoulos MA: Markers of bone remodeling and skeletal morbidity in patients with solid tumors metastatic to the skeleton receiving the biphosphonate zoledronic acid. Transl Res 155: 247-255, 2010.

16. Ulrich U, Rhiem K, Schmolling J, Flaskamp C, Paffenholz I, Sälzer H, Bauknecht T and Schlebusch H: Cross-linked type I collagen $\mathrm{C}$ - and $\mathrm{N}$-telopeptides in women with bone metastases from breast cancer. Arch Gynecol Obstet 264: 186-190, 2001.

17. Lipton A, Cook R, Saad F, Major P, Garnero P, Terpos E, Brown JE and Coleman RE: Normalization of bone markers is associated with improved survival in patients with bone metastases from solid tumors and elevated bone resorption receiving zoledronic acid. Cancer 113: 193-201, 2008.

18. Ibrahim T, Sacanna E, Gaudio M, Mercatali L, Scarpi E, Zoli W, Serra P, Ricci R, Serra L, Kang Y and Amadori D: Role of RANK, RANKL, OPG and CXCR4 tissue markers in predicting bone metastases in breast cancer patients. Clin Breast Cancer 11: 369-375, 2011.

19. Mercatali L, Ricci M, Scarpi E, Serra P, Fabbri F, Ricci R, Liverani C, Zanoni M, Zoli W, Maltoni R, et al: RANK/RANK-L/OPG in patients with bone metastases treated with anticancer agents and zoledronic acid: A prospective study. Int J Mol Sci 14: 10683-10693, 2013.

20. Ibrahim T, Flamini E, Mercatali L, Sacanna E, Serra P and Amadori D: Pathogenesis of osteoblastic bone metastases from prostate cancer. Cancer 116: 1406-1418, 2010.

21. Ibrahim T, Mercatali L and Amadori D: A new emergency in oncology: Bone metastases in breast cancer patients (Review). Oncol Lett 6: 306-310, 2013.

22. Drooger JC, van der Padt A, Sleijfer S and Jager A: Denosumab in breast cancer treatment. Eur J Pharmacol 717: 12-19, 2013.

23. Hayashi N, Costelloe CM, Hamaoka T, Wei C, Niikura N, Theriault RL, Hortobagyi GN, Madewell JE and Ueno NT: A prospective study of bone tumor response assessment in metastatic breast cancer. Clin Breast Cancer 13: 24-30, 2013.

24. Amadori D, Mercatali L, Nanni O, Aglietta M, Alessi B, Gianni L, Farina G, Gaion F, Bertoldo F, Santini D, et al: What can we learn from the ZOOM trial?-Authors' reply. Lancet Oncol 14: e388-e390, 2013.

25. Coleman RE, Major P, Lipton A, Brown JE, Lee KA, Smith M, Saad F, Zheng M, Hei YJ, Seaman J, et al: Predictive value of bone resorption and formation markers in cancer patients with bone metastases receiving the bisphosphonate zoledronic acid. J Clin Oncol 23: 4925-4935, 2005.

26. Brown JE, Cook RJ, Major P, Lipton A, Saad F, Smith M, Lee KA, Zheng M, Hei YJ and Coleman RE: Bone turnover markers as predictors of skeletal complications in prostate cancer, lung cancer and other solid tumors. J Natl Cancer Inst 97: 59-69, 2005 .

27. Coleman R, Costa L, Saad F, Cook R, Hadji P, Terpos E, Garnero P, Brown J, Body JJ, Smith M, et al: Consensus on the utility of bone markers in the malignant bone disease setting. Crit Rev Oncol Hematol 80: 411-432, 2011.

28. Weilbaecher KN, Guise TA and McCauley LK: Cancer to bone: A fatal attraction. Nat Rev Cancer 11: 411-425, 2011.

29. Tamiya M, Tokunaga S, Okada H, Suzuki H, Kobayashi M, Sasada S, Okamoto N, Morishita N, Matsuura Y, Miyamoto $\mathrm{N}$, et al: Prospective study of urinary and serum cross-linked N-telopeptide of type I collagen (NTx) for diagnosis of bone metastasis in patients with lung cancer. Clin Lung Cancer 14: 364-369, 2013. 